\title{
Solving Nonlinear Fourth-Order Boundary Value Problems Using a Numerical Approach: $(m+1)$ th-Step Block Method
}

\author{
Oluwaseun Adeyeye and Zurni Omar \\ Mathematics Department, School of Quantitative Sciences, Universiti Utara Malaysia, Sintok, Kedah, Malaysia \\ Correspondence should be addressed to Oluwaseun Adeyeye; adeyeye_oluwaseun@ahsgs.uum.edu.my
}

Received 15 June 2017; Revised 29 August 2017; Accepted 3 October 2017; Published 29 October 2017

Academic Editor: Davood D. Ganji

Copyright (C) 2017 Oluwaseun Adeyeye and Zurni Omar. This is an open access article distributed under the Creative Commons Attribution License, which permits unrestricted use, distribution, and reproduction in any medium, provided the original work is properly cited.

\begin{abstract}
Nonlinear boundary value problems (BVPs) are more tedious to solve than their linear counterparts. This is observed in the extra computation required when determining the missing conditions in transforming BVPs to initial value problems. Although a number of numerical approaches are already existent in literature to solve nonlinear BVPs, this article presents a new block method with improved accuracy to solve nonlinear BVPs. A $(m+1)$ th-step block method is developed using a modified Taylor series approach to directly solve fourth-order nonlinear boundary value problems (BVPs) where $m$ is the order of the differential equation under consideration. The schemes obtained were combined to simultaneously produce solution to the fourth-order nonlinear BVPs at $m+1$ points iteratively. The derived block method showed improved accuracy in comparison to previously existing authors when solving the same problems. In addition, the suitability of the $(m+1)$ th-step block method was displayed in the solution for magnetohydrodynamic squeezing flow in porous medium.
\end{abstract}

\section{Introduction}

Boundary value problems (BVPs) arise in several branches of science ranging from physical sciences to engineering. There has been commendable progress in solving problems associated with nonlinear ordinary differential equations (ODEs) involving boundary conditions in recent years. These ODEs are sometimes needed to fulfil certain boundary conditions at more than one point of the independent variable which will result in the problem known as two-point boundary value problem. Two-point nonlinear BVPs often cannot be solved by analytical methods and thus finding approximate solutions for these problems becomes essential.

This article considers the following special type of nonlinear boundary value problem:

$$
y^{i v}=f(x, y),
$$

with the boundary conditions

$$
\begin{aligned}
y(a) & =\alpha_{1}, \\
y^{\prime}(a) & =\alpha_{2}, \\
y(b) & =\beta_{1}, \\
y^{\prime}(b) & =\beta_{2},
\end{aligned}
$$

where $f$ is a continuous function on $[a, b]$ and the parameters $\alpha_{i}$ and $\beta_{i}$ for $i=1,2$ are constants.

A variety of methods have been introduced to solve (1) such as shooting methods, splines methods, finite difference methods, finite element methods, differential transform methods, and collocation methods [1-4]. Recently, the adoption of various families of linear multistep method (LMM) for numerically approximating higher order ODEs has been proposed. However, some LMMs cannot directly solve these higher order ODEs and thus require reduction to a system of first-order ODEs. In some cases, the accuracy of LMMs is low such as the case of predictor-corrector methods which incur high computational rigour. This computational rigour 
involves the derivation of separate predictors for each grid point of the LMM as seen in the work of Kayode and Adeyeye [5] and Kayode and Obarhua [6]. These drawbacks caused for the introduction of block methods which were first proposed by Milne [7] as a means to obtain starting values for predictorcorrector methods. This concept was also further explored by Sarafyan [8].

Block methods differ from alternate approaches such as differential transform method and collocation method. This is because the formulation of block methods is an evaluation of the linear multistep method at different grid points to generate a family of methods that can be applied to produce approximate solutions of ODEs at each grid point simultaneously. This advantage was mentioned by Lambert [9] among other advantages which include being self-starting, permitting easy change of step-length, and being less expensive in terms of function evaluations. Block methods also yield better accuracy when applied to numerical problems.

This article introduces a $(m+1)$ th-step block method where $m$ is the order of the differential equation. The block method is developed using a modification of the conventional Taylor series expansions approach by Lambert [9]. This derivation is shown in Section 2 of this article while Section 3 considers certain numerical examples and their results to show the accuracy of the block method.

\section{Methodology}

Lambert [9] highlighted three main approaches for developing LMMs. These include interpolation, numerical integration, and Taylor series expansions. This article adopts the Taylor series expansion approach for LMMs to develop the block method. However, certain modifications were introduced since Lambert [9] focused on first-order methods whereas this article develops a block method for fourthorder ODEs. Therefore, the approach was made suitable to develop block methods and not LMMs alone, hence the name modified Taylor series approach.

2.1. Derivation of the $(m+1)$ th-Step Block Method Using Modified Taylor Series Approach. The algorithm described below is used to show the steps involved in deriving the $(m+1)$ th-step block method using the modified Taylor series approach where $m$ is the order of the differential equation.

\section{Algorithm 1.}

Start.

Step 1. Obtain the coefficients of the initial multistep scheme:

$$
y_{n+(m+1)}=\sum_{j=(m-4)}^{m} \alpha_{j_{v}} y_{n+j}+\sum_{j=(m-4)}^{(m+1)} \beta_{j} f_{n+j},
$$

$v=1,2, \ldots, m$.
Step 2. Obtain the coefficients of the additional schemes:

$$
\begin{gathered}
y_{n+j_{m+1}}=\sum_{j=(m-4)}^{m} \alpha_{j_{v}} y_{n+j}+\sum_{j=(m-4)}^{(m+1)} \beta_{j} f_{n+j}, \\
y_{n+j_{m+2}}=\sum_{j=(m-4)}^{m} \alpha_{j_{v}} y_{n+j}+\sum_{j=(m-4)}^{(m+1)} \beta_{j} f_{n+j}, \\
\vdots \\
y_{n+j_{k}}=\sum_{j=(m-4)}^{m} \alpha_{j_{v}} y_{n+j}+\sum_{j=(m-4)}^{(m+1)} \beta_{j} f_{n+j} .
\end{gathered}
$$

Step 3. Derive the coefficients of the (1st, 2 nd, ..., $(m-1)$ th $)$ derivative schemes:

$$
\begin{gathered}
y_{n}^{(\varrho)}=\sum_{j=(m-4)}^{m} \alpha_{j_{v}} y_{n+j}+\sum_{j=(m-4)}^{(m+1)} \beta_{j} f_{n+j}, \\
y_{n+1}^{(\varrho)}=\sum_{j=(m-4)}^{m} \alpha_{j_{v}} y_{n+j}+\sum_{j=(m-4)}^{(m+1)} \beta_{j} f_{n+j}, \\
\vdots \\
y_{n+(m+1)}^{(\varrho)}=\sum_{j=(m-4)}^{m} \alpha_{j_{v}} y_{n+j}+\sum_{j=(m-4)}^{(m+1)} \beta_{j} f_{n+j},
\end{gathered}
$$

where $\varrho=1,2, \ldots, m-1$.

Step 4. Combine schemes obtained in Steps 1, 2, and 3 above to form a system of equations with matrix form equivalent $A x=B$ where $x=\left(N_{0}, N_{1}, N_{2}, \ldots, N_{m-1}\right)^{T}$ and $N_{0}=$ $\left(y_{n+(m-3)}, y_{n+(m-2)}, \ldots, y_{n+(m+1)}\right)^{T}, N_{1}=\left(y_{n+(m-3)}^{\prime}, y_{n+(m-2)}^{\prime}\right.$, $\left.\ldots, y_{n+(m+1)}^{\prime}\right)^{T}, \quad N_{2}=\left(y_{n+(m-3)}^{\prime \prime}, y_{n+(m-2)}^{\prime \prime}, \ldots, y_{n+(m+1)}^{\prime \prime}\right)^{T}$, $N_{m-1}=\left(y_{n+(m-3)}^{m-1}, y_{n+(m-2)}^{m-1}, \ldots, y_{n+(m+1)}^{m-1}\right)^{T}$

Step 5. Adopt matrix inverse approach to system of equations in Step 4 to obtain the expected block method.

Stop.

In Algorithm 1, $y_{n+a}=y\left(x_{n+a}\right)=y\left(x_{n}+a h\right), f_{n+j}=$ $f\left(x_{n+j}, y_{n+j}\right)$, and $\alpha_{j_{v}}$ and $\beta_{j}$ are constants with $v$ defined in Step 1 of Algorithm 1 .

Note that in Step 1 of Algorithm 1, the expected $\alpha_{j_{v}}$ are $\alpha_{j_{1}}, \alpha_{j_{2}}, \ldots, \alpha_{j_{m}}$. The $\alpha_{j_{v}}$-values can take ${ }^{k} C_{v}$ forms and $j_{v^{-}}$ values not chosen will be used as evaluation points when developing the additional methods in Step 2. 
Steps 1-3 of Algorithm 1 require expanding individual terms using Taylor Series expansion such as

$$
\begin{aligned}
& y_{n}=y\left(x_{n}\right), \\
& y_{n+1}=y\left(x_{n}+h\right)=y\left(x_{n}\right)+h y^{\prime}\left(x_{n}\right)+\frac{h^{2}}{2 !} y^{\prime \prime}\left(x_{n}\right) \\
& +\cdots, \\
& \quad \vdots \\
& y_{n+(k-1)}=y\left[x_{n}+((k-1) h)\right]=y\left(x_{n}\right) \\
& \quad+((k-1) h) y^{\prime}\left(x_{n}\right)+\frac{((k-1) h)^{2}}{2 !} y^{\prime \prime}\left(x_{n}\right)+\cdots, \\
& y_{n+k}=y\left[x_{n}+k h\right]=y\left(x_{n}\right)+(k h) y^{\prime}\left(x_{n}\right) \\
& \quad+\frac{(k h)^{2}}{2 !} y^{\prime \prime}\left(x_{n}\right)+\ldots, \\
& f_{n}=y^{(m)}\left(x_{n}\right), \\
& f_{n+1}=y^{(m)}\left(x_{n}+h\right)=y^{(m)}\left(x_{n}\right)+h y^{(m+1)}\left(x_{n}\right)
\end{aligned}
$$

$$
+\frac{h^{2}}{2 !} y^{(m+2)}\left(x_{n}\right)+\cdots,
$$

$$
\begin{aligned}
& f_{n+(k-1)}=y^{(m)}\left[x_{n}+((k-1) h)\right]=y^{(m)}\left(x_{n}\right) \\
& \quad+((k-1) h) y^{(m+1)}\left(x_{n}\right)+\frac{((k-1) h)^{2}}{2 !} y^{(m+2)}\left(x_{n}\right) \\
& \quad+\cdots, \\
& f_{n+k}=y^{(m)}\left[x_{n}+k h\right]=y^{(m)}\left(x_{n}\right)+(k h) y^{(m+1)}\left(x_{n}\right) \\
& \quad+\frac{(k h)^{2}}{2 !} y^{(m+2)}\left(x_{n}\right)+\cdots .
\end{aligned}
$$

Substituting these expansions in individual equations and equating coefficients of $y^{(m)} x_{n}$ presents the resulting expressions in matrix form

$$
A \mathbf{x}=\mathbf{B},
$$

where

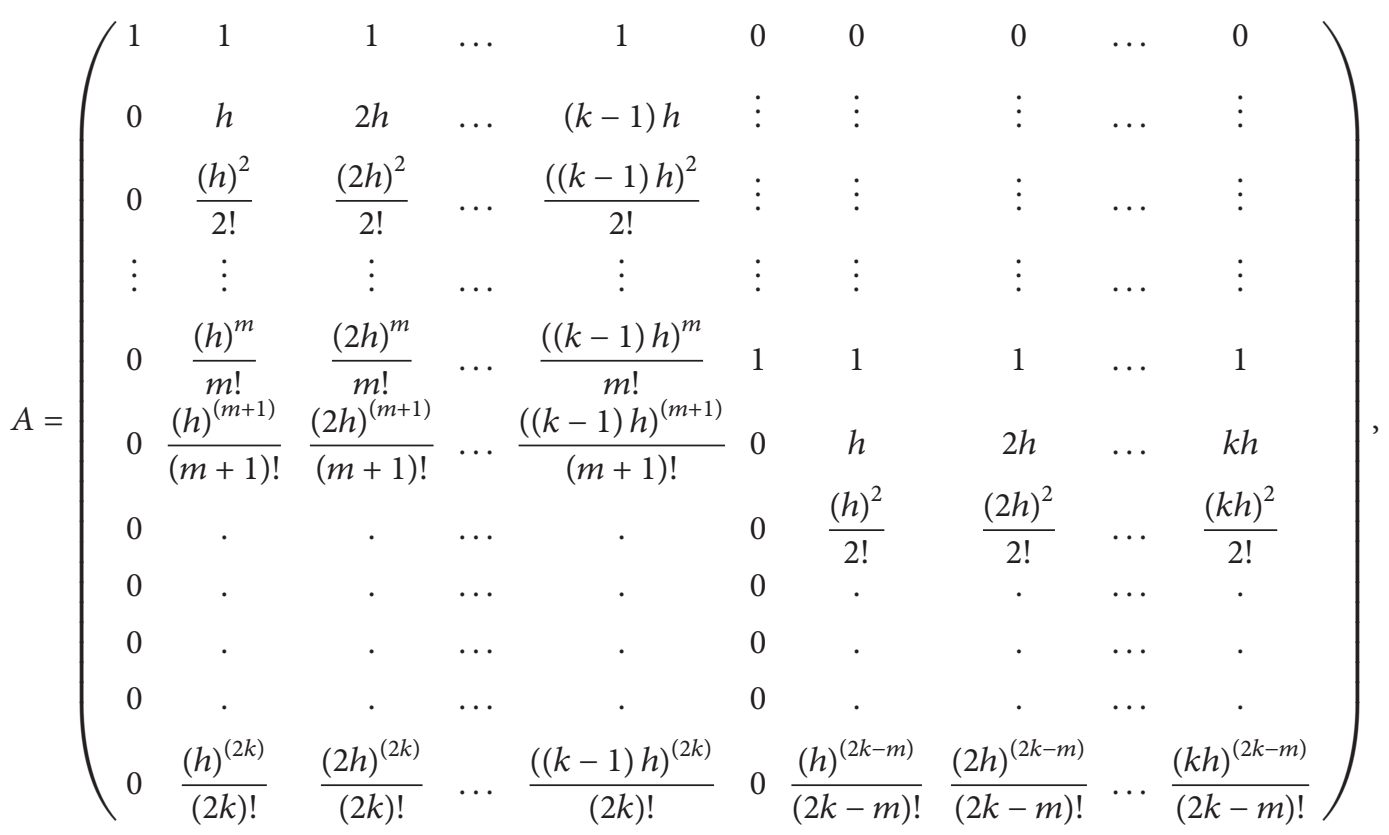

$$
\begin{aligned}
& \mathbf{x}=\left(\alpha_{0}, \alpha_{1}, \alpha_{2}, \ldots, \alpha_{k-1}, \beta_{0}, \beta_{1}, \beta_{2}, \ldots, \beta_{k}\right)^{T}, \\
& \mathbf{B}=\left(1, k h, \frac{(k h)^{2}}{2 !}, \frac{(k h)^{3}}{3 !}, \frac{(k h)^{4}}{4 !}, \ldots, \frac{(k h)^{(2 k)}}{(2 k) !}\right)^{T}
\end{aligned}
$$

Note that Algorithm 1 will not successfully obtain the required block method if matrix $A$ is singular. Thus, the nonsingularity of the resulting matrices is discussed.
2.2. Nonsingularity of Resulting Matrices. The matrix $A$ in (7) is a square matrix with $\operatorname{det}(A) \neq 0$ which follows from the theorems below. 
Theorem 2. Suppose that $A$ is a square matrix with a row where every entry is zero, or a column where every entry is zero. Then $\operatorname{det}(A)=0$.

Theorem 3. Suppose that $A$ is a square matrix with two equal rows, or two equal columns. Then $\operatorname{det}(A)=0$.

With respect to Theorem 2, since the matrix $A$ does not have a row or column where every entry is zero, then its inverse exists. On the other hand, matrix $A$ has no equal rows or columns which further affirms that its inverse exists.

Theorems 2 and 3 are sufficient conditions to show that the inverse of the resulting matrix will always exist. In addition, the case of linear dependency is considered as defined in the following theorems.

Theorem 4. If matrix $A$ has linearly dependent columns, then $\operatorname{det}(A)=0$.

Theorem 5. The rank of a matrix $A$ equals the maximum number of linearly independent column vectors. The matrix $A$ has the same number of linearly independent row vectors as it has linearly independent column vectors
Thus, Theorem 5 is tested for resulting matrices obtained in developing the $(m+1)$ th-step block method to show that $A^{-1}$ exists.

2.3. Specification of the $(m+1)$ th-Step Block Method. Following Algorithm 1, the specification of the $(m+1)$ th-step block method is as follows. From Step 1, the initial multistep scheme for the $(m+1)$ th-step block method in terms of $m$ is

$$
\begin{aligned}
y_{n+m+1}= & \alpha_{m-4} y_{n+m-4}+\alpha_{m-3} y_{n+m-3}+\alpha_{m-2} y_{n+m-2} \\
& +\alpha_{m-1} y_{n+m-1}+\alpha_{m} y_{n+m}+\beta_{m-4} f_{n+m-4} \\
& +\beta_{m-3} f_{n+m-3}+\beta_{m-2} f_{n+m-2}+\beta_{m-1} f_{n+m-1} \\
& +\beta_{m} f_{n+m}+\beta_{m+1} f_{n+m+1} .
\end{aligned}
$$

Now, considering (9), the individual terms are expanded using Taylor series expansion as defined in (6). The resulting expansions are substituted back in (9) and rewritten in matrix form $A \mathbf{x}=\mathbf{B}$, where

A

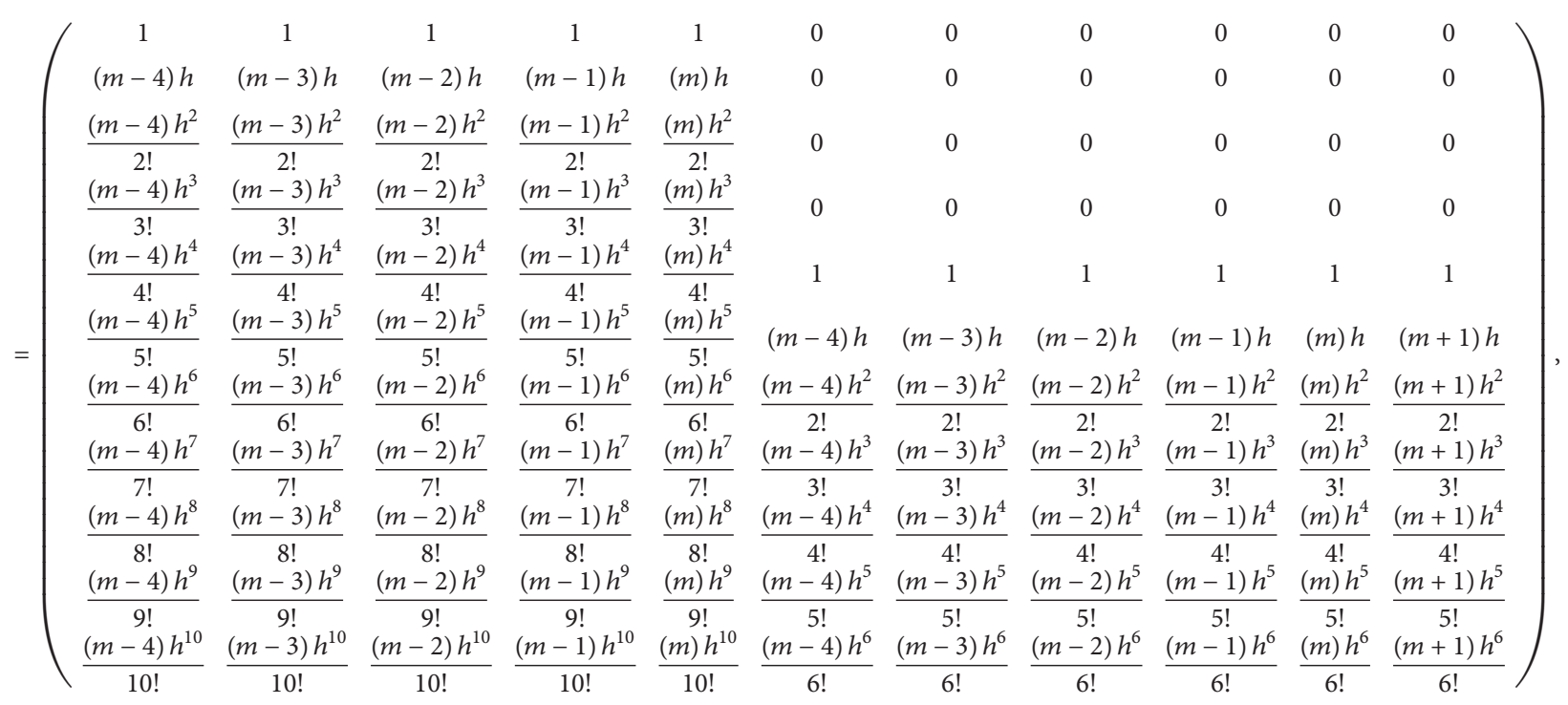

$\mathbf{x}=\left(\alpha_{m-4}, \alpha_{m-3}, \alpha_{m-2}, \alpha_{m-1}, \alpha_{m}, \beta_{m-4}, \beta_{m-3}, \beta_{m-2}, \beta_{m-1}, \beta_{m}, \beta_{m+1}\right)^{T}$,

B

$$
=\left(1,(m+1) h, \frac{((m+1) h)^{2}}{2 !}, \frac{((m+1) h)^{3}}{3 !}, \frac{((m+1) h)^{4}}{4 !}, \frac{((m+1) h)^{5}}{5 !}, \frac{((m+1) h)^{6}}{6 !}, \frac{((m+1) h)^{7}}{7 !}, \frac{((m+1) h)^{8}}{8 !}, \frac{((m+1) h)^{9}}{9 !}, \frac{((m+1) h)^{10}}{10 !}\right)^{T},
$$

where matrix $A$ has rank $=11$ which implies that there are no linearly dependent columns or rows and the inverse exists. This follows from the theorems in Section 2.2 showing that the matrix is nonsingular. Therefore, the scheme in (9) is obtained using matrix inverse method and substituting the value of $m$ as

$$
\begin{aligned}
& y_{n+m+1}=y_{n+m-4}-5 y_{n+m-3}+10 y_{n+m-2}-10 y_{n+m-1} \\
& \quad+5 y_{n+m}+\frac{h^{4}}{720}\left(f_{n+m-4}-125 f_{n+m-3}-350 f_{n+m-2}\right. \\
& \left.\quad+350 f_{n+m-1}+125 f_{n+m}+f_{n+m+1}\right) .
\end{aligned}
$$


Following the subsequent steps of Algorithm 1, the specification of the $(m+1)$ th-step block method is as follows:

$$
\begin{aligned}
& 1814400 y_{n+m-3}=1814400\left(y_{n}+h y_{n}^{\prime}+\frac{h^{2}}{2} y_{n}^{\prime \prime}\right. \\
& \left.+\frac{h^{3}}{6} y_{n}^{\prime \prime \prime}\right)+h^{4}\left(49126 f_{n}+49045 f_{n+m-3}\right. \\
& -40160 f_{n+m-2}+25430 f_{n+m-1}-9310 f_{n+m} \\
& \left.+1469 f_{n+m+1}\right) \text {, } \\
& 14175 y_{n+m-2}=14175\left(y_{n}+2 h y_{n}^{\prime}+2 h^{2} y_{n}^{\prime \prime}\right. \\
& \left.+\frac{4 h^{3}}{3} y_{n}^{\prime \prime \prime}\right)+h^{4}\left(4264 f_{n}+7960 f_{n+m-3}\right. \\
& -4910 f_{n+m-2}+3080 f_{n+m-1}-1120 f_{n+m} \\
& \left.+176 f_{n+m+1}\right) \\
& 22400 y_{n+m-1}=22400\left(y_{n}+3 h y_{n}^{\prime}+\frac{9 h^{2}}{2} y_{n}^{\prime \prime}\right. \\
& \left.+\frac{9 h^{3}}{2} y_{n}^{\prime \prime \prime}\right)+h^{4}\left(25488 f_{n}+63315 f_{n+m-3}\right. \\
& -26460 f_{n+m-2}+19170 f_{n+m-1}-7020 f_{n+m} \\
& \left.+1107 f_{n+m+1}\right) \text {, } \\
& 14175 y_{n+m}=14175\left(y_{n}+4 h y_{n}^{\prime}+8 h^{2} y_{n}^{\prime \prime}\right. \\
& \left.+\frac{32 h^{3}}{3} y_{n}^{\prime \prime \prime}\right)+h^{4}\left(40448 f_{n}+116480 f_{n+m-3}\right. \\
& -29440 f_{n+m-2}+33280 f_{n+m-1}-11360 f_{n+m} \\
& \left.+1792 f_{n+m+1}\right) \text {, }
\end{aligned}
$$

$$
\begin{aligned}
& y_{n+m+1}=y_{n}+5 h y_{n}^{\prime}+\frac{25 h^{2}}{2} y_{n}^{\prime \prime}+\frac{125 h^{3}}{6} y_{n}^{\prime \prime \prime} \\
& +\frac{h^{4}}{72576}\left(418250 f_{n}+1315625 f_{n+m-3}\right. \\
& -175000 f_{n+m-2}+418750 f_{n+m-1}-106250 f_{n+m} \\
& \left.+18625 f_{n+m+1}\right),
\end{aligned}
$$

$$
\begin{aligned}
& 40320 y_{n+m-3}^{\prime}=40320\left(y_{n}^{\prime}+h y_{n}^{\prime \prime}+\frac{h^{2}}{2} y_{n}^{\prime \prime \prime}\right) \\
& +h^{3}\left(3929 f_{n}+4975 f_{n+m-3}-3862 f_{n+m-2}\right. \\
& \left.+2422 f_{n+m-1}-883 f_{n+m}+139 f_{n+m+1}\right), \\
& 630 y_{n+m-2}^{\prime}=630\left(y_{n}^{\prime}+2 h y_{n}^{\prime \prime}+2 h^{2} y_{n}^{\prime \prime \prime}\right)+h^{3}\left(317 f_{n}\right.
\end{aligned}
$$

$$
\begin{aligned}
& +734 f_{n+m-3}-380 f_{n+m-2}+244 f_{n+m-1}-89 f_{n+m} \\
& \left.+14 f_{n+m+1}\right)
\end{aligned}
$$$$
4480 y_{n+m-1}^{\prime}=4480\left(y_{n}^{\prime}+3 h y_{n}^{\prime \prime}+\frac{9 h^{2}}{2} y_{n}^{\prime \prime \prime}\right)
$$$$
+h^{3}\left(5481 f_{n}+16119 f_{n+m-3}-4374 f_{n+m-2}\right.
$$$$
\left.+4230 f_{n+m-1}-1539 f_{n+m}+243 f_{n+m+1}\right),
$$$$
315 y_{n+m}^{\prime}=315\left(y_{n}^{\prime}+4 h y_{n}^{\prime \prime}+8 h^{2} y_{n}^{\prime \prime \prime}\right)+h^{3}\left(712 f_{n}\right.
$$$$
+2336 f_{n+m-3}-224 f_{n+m-2}+704 f_{n+m-1}
$$$$
\left.-200 f_{n+m}+32 f_{n+m+1}\right) \text {, }
$$

$$
\begin{aligned}
& 8064 y_{n+m+1}^{\prime}=8064\left(y_{n}^{\prime}+5 h y_{n}^{\prime \prime}+\frac{25 h^{2}}{2} y_{n}^{\prime \prime \prime}\right) \\
& +h^{3}\left(29125 f_{n}+101875 f_{n+m-3}+1250 f_{n+m-2}\right. \\
& \left.\quad+38750 f_{n+m-1}-4375 f_{n+m}+1375 f_{n+m+1}\right)
\end{aligned}
$$

$$
\begin{aligned}
& 10080 y_{n+m-3}^{\prime \prime}=10080\left(y_{n}^{\prime \prime}+h y_{n}^{\prime \prime \prime}\right)+h^{2}\left(2462 f_{n}\right. \\
& +4315 f_{n+m-3}-3044 f_{n+m-2}+1882 f_{n+m-1} \\
& \left.-682 f_{n+m}+107 f_{n+m+1}\right) \text {, } \\
& 630 y_{n+m-2}^{\prime \prime}=630\left(y_{n}^{\prime \prime}+2 h y_{n}^{\prime \prime \prime}\right)+h^{2}\left(355 f_{n}\right. \\
& +1088 f_{n+m-3}-370 f_{n+m-2}+272 f_{n+m-1} \\
& \left.-101 f_{n+m}+16 f_{n+m+1}\right) \text {, } \\
& 1120 y_{n+m-1}^{\prime \prime}=1120\left(y_{n}^{\prime \prime}+3 h y_{n}^{\prime \prime \prime}\right)+h^{2}\left(984 f_{n}\right. \\
& +3501 f_{n+m-3}-72 f_{n+m-2}+870 f_{n+m-1}-288 f_{n+m} \\
& \left.+45 f_{n+m+1}\right) \text {, } \\
& 315 y_{n+m}^{\prime \prime}=315\left(y_{n}^{\prime \prime}+4 h y_{n}^{\prime \prime \prime}\right)+h^{2}\left(376 f_{n}\right. \\
& +1424 f_{n+m-3}+176 f_{n+m-2}+608 f_{n+m-1}-80 f_{n+m} \\
& \left.+16 f_{n+m+1}\right) \text {, } \\
& 2016 y_{n+m+1}^{\prime \prime}=2016\left(y_{n}^{\prime \prime}+5 h y_{n}^{\prime \prime \prime}\right)+h^{2}\left(3050 f_{n}\right. \\
& +11875 f_{n+m-3}+2500 f_{n+m-2}+6250 f_{n+m-1} \\
& \left.+1250 f_{n+m}+275 f_{n+m+1}\right) \\
& 1440 y_{n+1}^{\prime \prime \prime}=1440 y_{n}^{\prime \prime \prime}+h\left(475 f_{n}+1427 f_{n+m-3}\right. \\
& \left.-798 f_{n+m-2}+482 f_{n+m-1}-173 f_{n+m}+27 f_{n+m+1}\right) \text {, } \\
& 90 y_{n+2}^{\prime \prime \prime}=90 y_{n}^{\prime \prime \prime}+h\left(28 f_{n}+129 f_{n+m-3}+14 f_{n+m-2}\right. \\
& \left.+14 f_{n+m-1}-6 f_{n+m}+f_{n+m+1}\right),
\end{aligned}
$$




$$
\begin{aligned}
& 160 y_{n+3}^{\prime \prime \prime}=160 y_{n}^{\prime \prime \prime}+h\left(51 f_{n}+219 f_{n+m-3}\right. \\
& \left.+114 f_{n+m-2}+114 f_{n+m-1}-21 f_{n+m}+3 f_{n+m+1}\right), \\
& 45 y_{n+4}^{\prime \prime \prime}=45 y_{n}^{\prime \prime \prime}+h\left(14 f_{n}+64 f_{n+m-3}+24 f_{n+m-2}\right. \\
& \left.\quad+64 f_{n+m-1}+14 f_{n+m}\right), \\
& 288 y_{n+5}^{\prime \prime \prime}=288 y_{n}^{\prime \prime \prime}+h\left(95 f_{n}+375 f_{n+m-3}\right. \\
& \left.+250 f_{n+m-2}+250 f_{n+m-1}+375 f_{n+m}+95 f_{n+m+1}\right) .
\end{aligned}
$$

2.4. Order and Stability Properties of the $(m+1)$ th-Step Block Method. To ensure convergence of the block method, its consistency and zero-stability need to be investigated. This follows from Fatunla (1988) which states that a linear multistep method is convergent iff it is consistent and zerostable.

Starting with the consistency property, a linear multistep method is consistent if it has order $p \geq 1$. Thus, the order of the $(m+1)$ th-step block method is investigated.

With reference to the definition in Lambert [9], Henrici [11], and Butcher [12], the order and error constant of the $(m+$ 1)th-step block method follow Definition 6.

Definition 6. The linear operator associated with LMM is defined as

$$
L[y(x) ; h]=\sum_{j=0}^{k}\left[\alpha_{j} y_{n+j}-\beta_{j} f_{n+j}\right] .
$$

On expanding $y_{n+j}$ and $f_{n+j}$ to obtain

$$
\begin{aligned}
L[y(x) ; h]= & C_{0} y\left(x_{n}\right)+C_{1} h y^{\prime}\left(x_{n}\right)+\cdots \\
& +C_{q} h^{q} y^{(q)}\left(x_{n}\right)+\cdots,
\end{aligned}
$$

where

$$
\begin{aligned}
& C_{0}=\alpha_{0}+\alpha_{1}+\alpha_{2}+\cdots+\alpha_{k}, \\
& C_{1}=\alpha_{1}+2 \alpha_{2}+\cdots+k \alpha_{k}, \vdots \\
& C_{q}=\frac{1}{q !}\left(\alpha_{1}+2^{q} \alpha_{2}+\cdots+k^{q} \alpha_{k}\right) \\
&-\frac{1}{(q-m) !}\left(\beta_{1}+2^{q-m} \beta_{2}+\cdots+k^{q-m} \beta_{k}\right), \\
& \quad q=2,3, \ldots,
\end{aligned}
$$

the method is said to be of order $p$ if $C_{0}=C_{1}=\cdots=C_{p}=$ $C_{p+1}=\cdots=C_{p+(m-1)}=0, C_{p+m} \neq 0$ and $C_{p+m}$ is the error constant.

The integrators of the block method (12) are of order six methods with the error constants, $C_{10}$ obtained as $2323 / 3628800,137 / 14175,1737 / 44800,1408 / 14175$, and $29375 / 145152$, respectively. Having order $p>1$, the consistency of the block method is affirmed.
Moving on to the second criterion for convergence which is the zero-stability of the block method. Note that this is the most important stability property a good numerical method should possess as it ensures convergence. The key word "zero" is based on the stability phenomenon in terms of convergence in the limit as step-size $(h)$ tends to zero.

Therefore, to test the zero-stability of the $(m+1)$ th-step block method, the integrators are normalized to give the first characteristic polynomial $\rho(r)$ as

$$
\rho(r)=\operatorname{det}\left(r A^{0}-A^{1}\right)=r^{m}(r-1),
$$

with $A^{0}=5 \times 5$ identity matrix

$$
A^{1}=\left(\begin{array}{lllll}
0 & 0 & 0 & 0 & 1 \\
0 & 0 & 0 & 0 & 1 \\
0 & 0 & 0 & 0 & 1 \\
0 & 0 & 0 & 0 & 1 \\
0 & 0 & 0 & 0 & 1
\end{array}\right) .
$$

The roots of $\rho(r)=0$ satisfy $\left|r_{j}\right| \leq 1$. Hence, the $(m+1)$ th-step block method is zero-stable.

\section{Results and Discussion}

This section tests the $(m+1)$ th-step block method on some nonlinear problems. The numerical results are shown in Tables 1-3 and Figures 1-3.

Example 7. Consider the following nonlinear boundary value problem [10]:

$$
y^{i v}-6 \exp (-4 y)=-12(1+x)^{4},
$$

with boundary conditions

$$
\begin{aligned}
& y(0)=0, \\
& y^{\prime}(0)=1, \\
& y(1)=\ln (2)=y^{\prime}(1)=0.5 .
\end{aligned}
$$

The exact solution of Example 7 is $y=\ln (1+x)$. The obtained numerical results for this problem are presented in Table 1 with $h=10^{-1}$. The maximum absolute error obtained by the $(m+1)$ th-step block method is $3.84959 \times 10^{-7}$ which is more accurate than the maximum error of $1.78 \times 10^{-3}$ by Mustafa et al. [10]. The graphical comparison between exact and computed solution is shown in Figure 1.

Example 8. Consider the following nonlinear boundary value problem [10]:

$$
\begin{aligned}
y^{i v}= & y^{2}-x^{10}+-4 x^{8}-4 x^{7}+8 x^{6}-4 x^{4}+120 x \\
& -48
\end{aligned}
$$

with boundary conditions

$$
\begin{aligned}
& y(0)=y^{\prime}(0)=0, \\
& y(1)=y^{\prime}(1)=1 .
\end{aligned}
$$


TABLE 1: Comparison of the $(m+1)$ th-step block method with Mustafa et al. [10] for solving Example 7.

\begin{tabular}{lcccc}
\hline$x$ & Exact solution & Computed solution & Error $[10]$ & Error $((m+1)$ th-step block method $)$ \\
\hline 0.0 & 0.00000000000 & 0.00000000000 & $0.000000 e+00$ & $0.000000 e+00$ \\
0.1 & 0.09531017980 & 0.09531018728 & 0.0002954265 & $7.472134 e-09$ \\
0.2 & 0.18232155679 & 0.18232159067 & 0.0008719341 & $3.387990 e-08$ \\
0.3 & 0.26236426447 & 0.26236434273 & 0.0014096072 & $7.826736 e-08$ \\
0.4 & 0.33647223662 & 0.33647237832 & 0.0017352146 & $1.417001 e-07$ \\
0.5 & 0.40546510811 & 0.40546533420 & 0.0017810699 & $2.260955 e-07$ \\
0.6 & 0.47000362925 & 0.47000394905 & 0.0015577013 & $3.198013 e-07$ \\
0.7 & 0.53062825106 & 0.53062863602 & 0.0011349902 & $3.849590 e-07$ \\
0.8 & 0.58778666490 & 0.58778704558 & 0.0006286279 & $3.806745 e-07$ \\
0.9 & 0.64185388617 & 0.64185415218 & 0.0001902154 & $2.660067 e-07$ \\
1.0 & 0.69314718056 & 0.69314718056 & $0.000000 e+00$ & $0.000000 e+00$ \\
\hline
\end{tabular}

TABLE 2: Comparison of the exact and computed solution of Example 8.

\begin{tabular}{|c|c|c|c|c|}
\hline$x$ & Exact solution & Computed solution & Error [10] & Error $((m+1)$ th-step block method $)$ \\
\hline 0.0 & 0.00000000000 & 0.00000000000 & $0.000000 e+00$ & $0.000000 e+00$ \\
\hline 0.1 & 0.01981000000 & 0.01981000000 & 0.0004095 & $0.000000 e+00$ \\
\hline 0.2 & 0.07712000000 & 0.07712000000 & 0.0025752 & $0.000000 e+00$ \\
\hline 0.3 & 0.16623000000 & 0.16623000000 & 0.0066432 & $0.000000 e+00$ \\
\hline 0.4 & 0.27904000000 & 0.27904000000 & 0.0115595 & $0.000000 e+00$ \\
\hline 0.5 & 0.40625000000 & 0.40625000000 & 0.0156708 & $0.000000 e+00$ \\
\hline 0.6 & 0.53856000000 & 0.53856000000 & 0.0173246 & $0.000000 e+00$ \\
\hline 0.7 & 0.66787000000 & 0.66787000000 & 0.0154706 & $0.000000 e+00$ \\
\hline 0.8 & 0.78848000000 & 0.78848000000 & 0.0102612 & $0.000000 e+00$ \\
\hline 0.9 & 0.89829000000 & 0.89829000000 & 0.0036517 & $0.000000 e+00$ \\
\hline 1.0 & 1.00000000000 & 1.00000000000 & $0.000000 e+00$ & $0.000000 e+00$ \\
\hline
\end{tabular}

TABLE 3: Absolute errors of fifth-order HAM and $(m+1)$ th-step block method when $R_{m p}=0$.

\begin{tabular}{lccc}
\hline$z$ & Exact solution & Error [13] & Error $((m+1)$ th-step block method $)$ \\
\hline 0.0 & 0 & 0 & 0 \\
0.1 & 0.085233703438701791 & $7.58785506649317 \times 10^{-10}$ & $4.032885 \times 10^{-14}$ \\
0.2 & 0.171320454429454980 & $1.39478356642186 \times 10^{-9}$ & $2.363387 \times 10^{-13}$ \\
0.3 & 0.259121838110931650 & $1.80948145356296 \times 10^{-9}$ & $6.848411 \times 10^{-13}$ \\
0.4 & 0.349516600242079760 & $1.94815263920844 \times 10^{-9}$ & $1.489975 \times 10^{-12}$ \\
0.5 & 0.443409441985037010 & $1.81075676675135 \times 10^{-9}$ & $2.768896 \times 10^{-12}$ \\
0.6 & 0.541740074458440520 & $1.45204859247627 \times 10^{-9}$ & $4.502620 \times 10^{-12}$ \\
0.7 & 0.645492623682151550 & $9.70818092582703 \times 10^{-10}$ & $6.029954 \times 10^{-12}$ \\
0.8 & 0.755705480041236500 & $4.90335771985428 \times 10^{-10}$ & $6.408873 \times 10^{-12}$ \\
0.9 & 0.873481690845957730 & $1.33847599670389 \times 10^{-10}$ & $4.708123 \times 10^{-12}$ \\
1.0 & 1.000000000000000000 & $2.22044604925031 \times 10^{-16}$ & $0.000000 \times 10^{0}$ \\
\hline
\end{tabular}

The exact solution of Example 8 is $y=x^{5}-2 x^{4}+2 x^{2}$. The obtained numerical results for this problem are presented in Table 2 with $h=10^{-1}$. The $(m+1)$ th-step block method gives precise and accurate results as the exact solution. This is far more encouraging than the maximum error of $1.73 \times 10^{-2}$ by Mustafa et al. [10]. The graphical comparison between exact and computed solution is also shown in Figure 2.

Examples 7 and 8 considered the solution of nonlinear boundary value problems solved by Mustafa et al. [10]. In their work, the authors adopted a numerical approach based on subdivision schemes. Although their approach gave good results, the $(m+1)$ th-step block method gave better results in terms of accuracy. This superiority in accuracy of the $(m+1)$ th-step block method is resultant from its self-starting implementation approach instead of the approach of Mustafa et al. [10] requiring choosing different subdivision schemes with certain adjustment of boundary conditions. The selfstarting approach of the block method requires no starting values which could reduce the accuracy of the method 


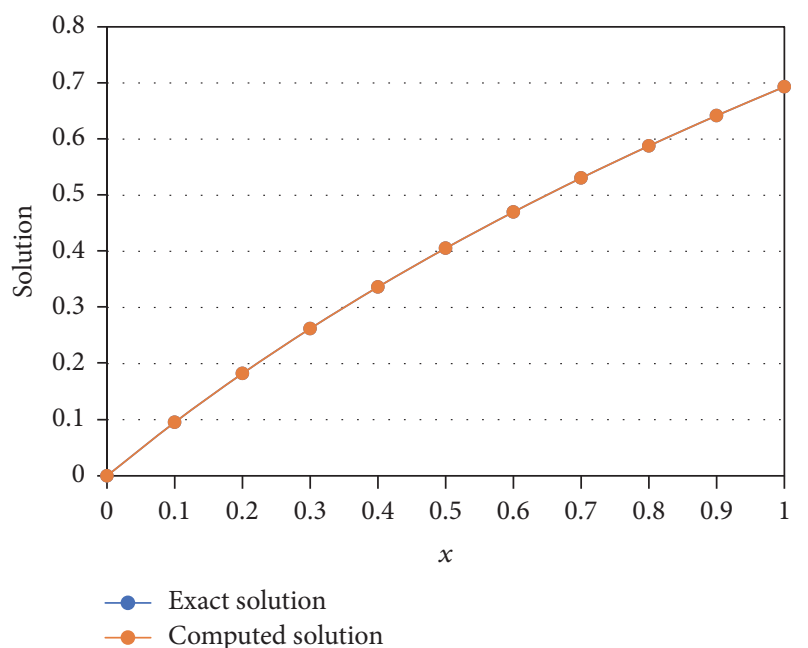

Figure 1: Comparison of the exact and computed solution of Example 7.

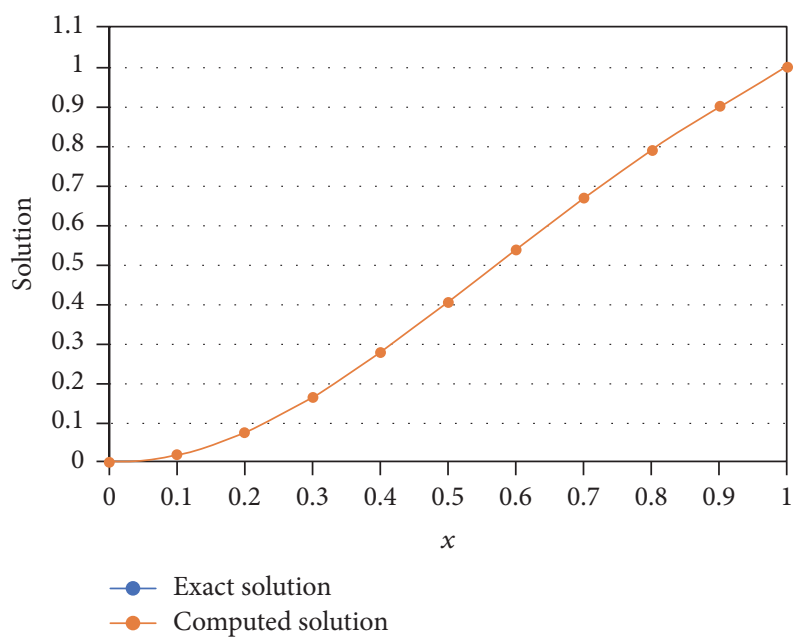

FIgURE 2: Comparison of the exact and computed solution of Example 8.

while also increasing the computational rigour. Rather, the integrators of $(m+1)$ th-step block method were combined as direct simultaneous integrators for the solution of the nonlinear boundary value problems.

In addition, to further show the suitability of the $(m+1)$ thstep block method, a physical problem is solved and the results are compared to exiting solutions in literature.

3.1. $(m+1)$ th-Step Block Method Solution for Magnetohydrodynamic Squeezing Flow in Porous Medium. The study of squeezing effect, in addition to other properties such as magnetohydrodynamics (MHD) and porosity, has become one of the most active topics in fluid mechanics. Ullah et al. [13] made an effort to investigate MHD squeezing flow of Newtonian fluid between two parallel plates passing through porous medium by homotopy analysis method (HAM). The authors used similarity transforms to convert the governing

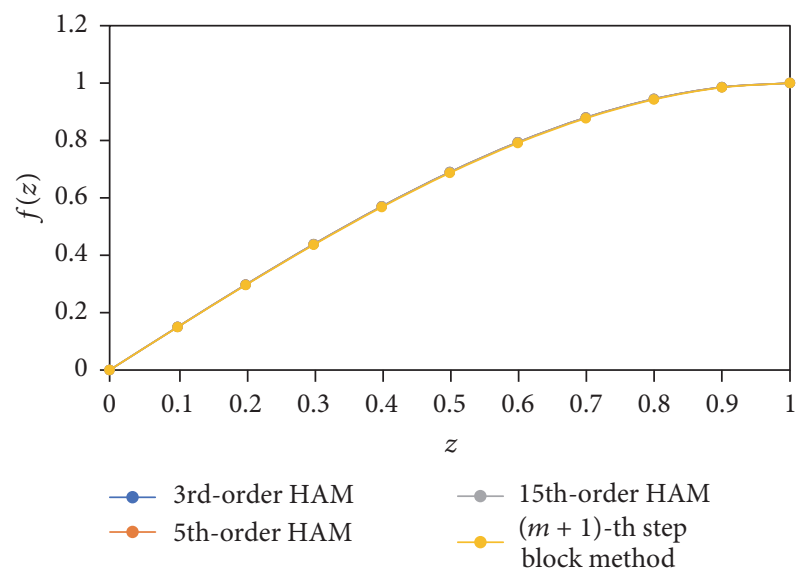

Figure 3: Comparison of solution between HAM [13] and $(m+1)$ thstep block method.

partial differential equations to equivalent nonlinear boundary value problems and then solved by HAM. This article takes a step further to solve the resulting nonlinear boundary value problems using the $(m+1)$ th-step block method.

The resulting differential equation is a fourth-order nonlinear boundary value problem of the form

$$
\begin{gathered}
\frac{d^{4}}{d z^{4}} f(z)+R_{m p} f(z) \frac{d^{3}}{d z^{3}} f(z)-m_{p} \frac{d^{2}}{d z^{2}} f(z) \\
-m_{h} \frac{d^{2}}{d z^{2}} f(z)=0
\end{gathered}
$$

with boundary conditions

$$
\begin{aligned}
\frac{d^{2}}{d z^{2}} f(0) & =0, \\
\frac{d}{d z} f(1) & =0, \\
f(0) & =0, \\
f(1) & =1,
\end{aligned}
$$

where $R_{m p}$ is Reynold number and $m_{h}, m_{p}$ are Hartmann numbers.

Due to the difficulty to compute an exact solution for (25), Ullah et al. [13] computed varying solutions of (25) for different HAM orders. The $(m+1)$ th-step block method is likewise adopted to solve (25) and convergence is observed to the results proposed by Ullah et al. [13] as seen in Figure 3.

In addition, a special case of (25) is studied when the Reynold number is zero with exact solution obtained using the boundary conditions (26) as

$$
\begin{aligned}
& f(z) \\
& =\frac{e^{2(-\sqrt{M} x)} e^{\sqrt{M}}-e^{\sqrt{M}}+\sqrt{M} x e^{-\sqrt{M} x}+\sqrt{M} x e^{-\sqrt{M} x} e^{2 \sqrt{M}}}{e^{-\sqrt{M} x}\left(\sqrt{M} e^{2 \sqrt{M}}-e^{2 \sqrt{M}}+\sqrt{M}+1\right)},
\end{aligned}
$$

where $M=m_{h}+m_{p}$. 
Comparison is made between the exact solution, fifthorder HAM solution [13], and the $(m+1)$ th-step block method in terms of absolute error.

From Table 3, an improved accuracy was displayed by the $(m+1)$ th-step block method over the homotopy analysis method. This shows the block method is appropriate to evaluate the numerical solution of physical problems modelled as fourth-order nonlinear boundary value problems.

\section{Conclusion}

This article has introduced a numerical approach based on block methods derived using modified Taylor series approach. The $(m+1)$ th-step block method was adopted for the numerical solution of different nonlinear fourthorder boundary value problems. The numerical results show that the impressive accuracy of the $(m+1)$ th-step block method having the same computations as the exact solution is obtained as shown in Tables 1-3 and Figures 1-3. This grounds the suitability of the $(m+1)$ th-step block method for solving fourth-order nonlinear boundary value problems. In addition, the suitability of the block method in application to physical problems was also investigated by presenting a solution to MHD squeezing flow in a porous medium. Convergence in solution and improved accuracy were properties displayed by the $(m+1)$ th-step block method when solving this fluid model. Thus, the $(m+1)$ th-step block method is appropriate for solving fourth-order nonlinear boundary value problems.

\section{Conflicts of Interest}

The authors declare that they have no conflicts of interest.

\section{Authors' Contributions}

Both authors contributed equally to this work.

\section{Acknowledgments}

This work is supported by Research and Innovation Management Centre (RIMC), Universiti Utara Malaysia.

\section{References}

[1] M. Abbas, A. A. Majid, A. I. Ismail, and A. Rashid, "The application of cubic trigonometric B-spline to the numerical solution of the hyperbolic problems," Applied Mathematics and Computation, vol. 239, pp. 74-88, 2014.

[2] L. Ahmad Soltani, E. Shivanian, and R. Ezzati, "Shooting homotopy analysis method: a fast method to find multiple solutions of nonlinear boundary value problems arising in fluid mechanics," Engineering Computations, vol. 34, no. 2, 2017.

[3] N. Freidoonimehr, M. M. Rashidi, and S. Mahmud, "Unsteady MHD free convective flow past a permeable stretching vertical surface in a nano-fluid," International Journal of Thermal Sciences, vol. 87, pp. 136-145, 2015.
[4] Z. Omar and J. O. Kuboye, "New seven-step numerical method for direct solution of fourth order ordinary differential equations," Journal of Mathematical and Fundamental Sciences, vol. 48, no. 2, pp. 94-105, 2016.

[5] S. J. Kayode and O. Adeyeye, "A 3-Step hybrid method for direct solution of second order initial value problems," Australian Journal of Basic and Applied Sciences, vol. 5, no. 12, pp. 21212126, 2011.

[6] S. J. Kayode and F. O. Obarhua, "Continuous $y$-function hybrid methods for direct solution of differential equations," International Journal of Differential Equations and Applications, vol. 12, no. 1, pp. 37-48, 2013.

[7] W. E. Milne, Numerical Solution of Ordinary Differential Equations, Wiley, New York, NY, USA, 1953.

[8] D. Sarafyan, "Multistep methods for the numerical solution of ordinary differential equations made self-starting," Mathematics Research Center, MRC-TSR-495, 1965.

[9] J. D. Lambert, Computational Methods in Ordinary Differential Equations, Wiley, New York, NY, USA, 1973.

[10] G. Mustafa, M. Abbas, S. T. Ejaz, A. I. M. Ismail, and F. Khan, "A numerical approach based on subdivision schemes for solving non-linear fourth order boundary value problems," Journal of Computational Analysis and Applications, vol. 23, no. 4, pp. 607623, 2017.

[11] P. Henrici, Discrete Variable Methods in Ordinary Differential Equations, John Wiley and Sons, New York, NY, USA, 1962.

[12] J. C. Butcher, Numerical Methods for Ordinary Differential Equations, Wiley, West Sussex, 2nd edition, 2008.

[13] I. Ullah, M. T. Rahim, H. Khan, and M. Qayyum, "Homotopy analysis solution for magnetohydrodynamic squeezing flow in porous medium," Advances in Mathematical Physics, vol. 2016, Article ID 3541512, 9 pages, 2016. 


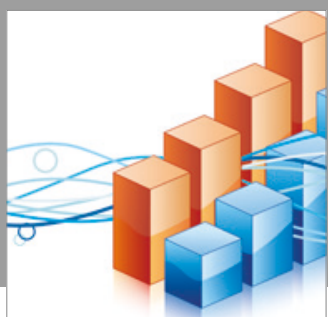

Advances in

Operations Research

vatersals

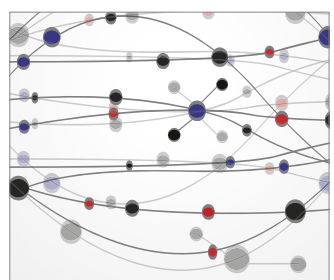

\section{The Scientific} World Journal
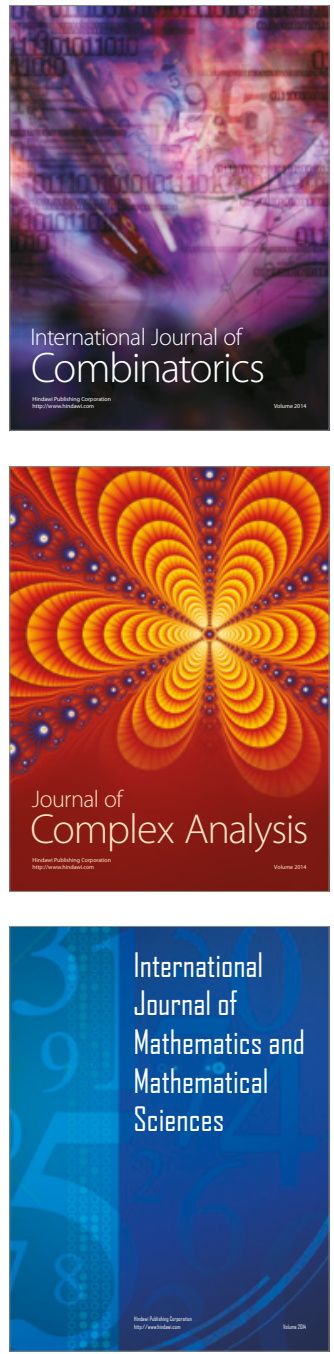
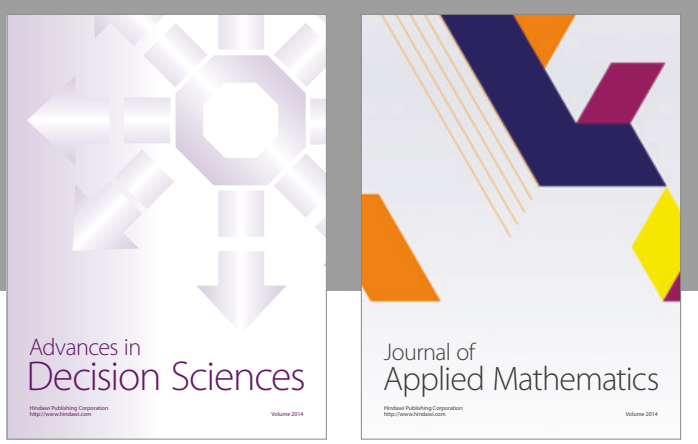

Algebra

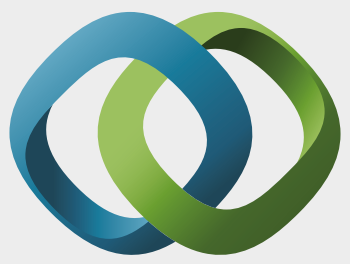

\section{Hindawi}

Submit your manuscripts at

https://www.hindawi.com
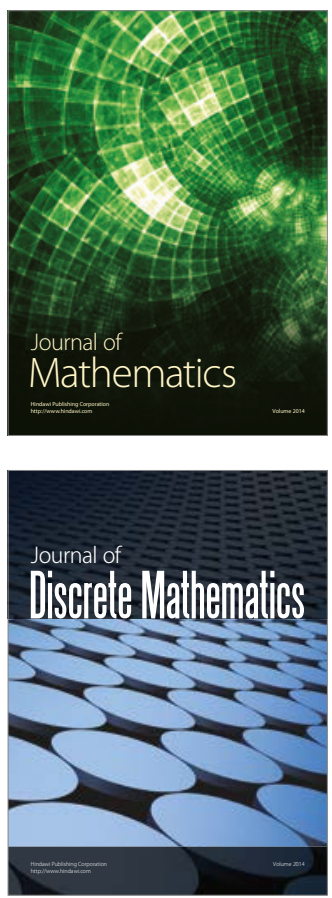

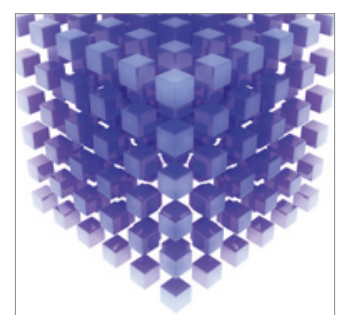

Mathematical Problems in Engineering
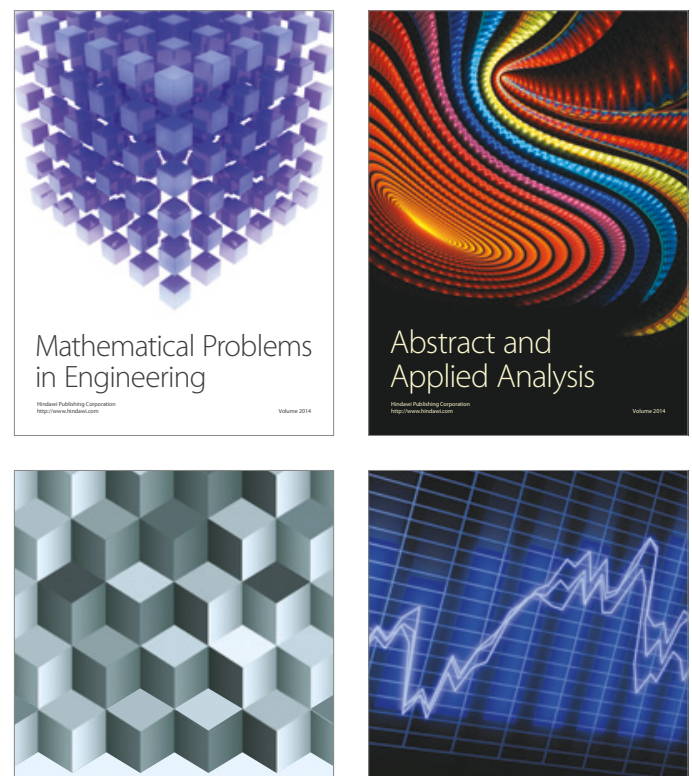

Journal of

Function Spaces

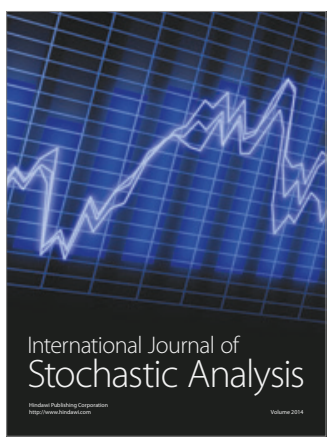

Probability and Statistics
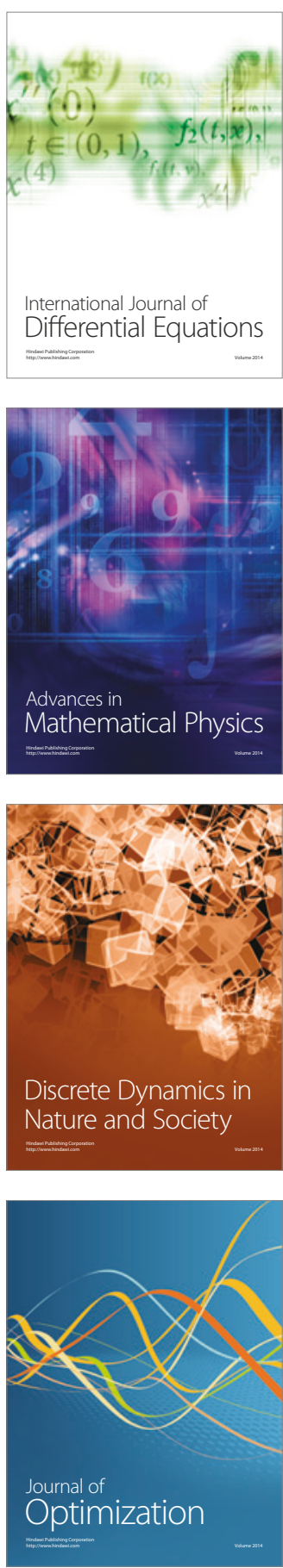